\title{
Rieger, Barbara
}

\section{Essgenuss im Jugendalter}

Haushalt in Bildung \& Forschung 6 (2017) 1, S. 62-78

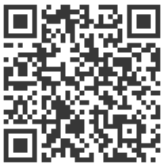

Quellenangabe/ Reference:

Rieger, Barbara: Essgenuss im Jugendalter - In: Haushalt in Bildung \& Forschung 6 (2017) 1, S. $62-78$ URN: urn:nbn:de:0111-pedocs-191329 - DOI: 10.25656/01:19132

https://nbn-resolving.org/urn:nbn:de:0111-pedocs-191329

https://doi.org/10.25656/01:19132

in Kooperation mit / in cooperation with:

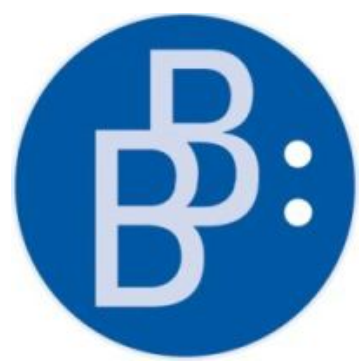

https://www.budrich.de

\section{Nutzungsbedingungen}

Gewährt wird ein nicht exklusives, nicht übertragbares, persönliches und beschränktes Recht auf Nutzung dieses Dokuments. Dieses Dokument ist ausschließlich für den persönlichen, nicht-kommerziellen Gebrauch bestimmt. Die Nutzung stellt keine Übertragung des Eigentumsrechts an diesem Dokument dar und gilt vorbehaltlich der folgenden Einschränkungen: Auf sämtlichen Kopien dieses Dokuments müssen alle Urheberrechtshinweise und sonstigen Hinweise auf gesetzlichen Schutz beibehalten werden. Sie dürfen dieses Dokument nicht in irgendeiner Weise abändern, noch dürfen Sie dieses Dokument für öffentliche oder kommerzielle Zwecke vervielfältigen, öffentlich ausstellen, aufführen, vertreiben oder anderweitig nutzen.

Mit der Verwendung dieses Dokuments erkennen Sie die Nutzungsbedingungen an.

\section{Terms of use}

We grant a non-exclusive, non-transferable, individual and limited right to using this document.

This document is solely intended for your personal, non-commercial use. Use of this document does not include any transfer of property rights and it is conditional to the following limitations: All of the copies of this documents must retain all copyright information and other information regarding legal protection. You are not allowed to alter this document in any way, to copy it for public or commercial purposes, to exhibit the document in public, to perform, distribute or otherwise use the document in public.

By using this particular document, you accept the above-stated conditions of use.

\section{Kontakt / Contact:}

\section{peDOcs}

DIPF | Leibniz-Institut für Bildungsforschung und Bildungsinformation Informationszentrum (IZ) Bildung

E-Mail:pedocs@dipf.de

Internet: www.pedocs.de

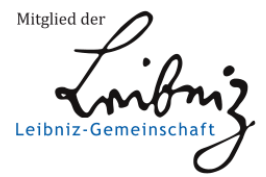


6. Jahrgang Heft 1

2017

峁

仓

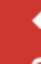

()

음

닌

亲

c

ชิ

은

\)

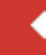

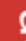

8

垔

ह

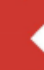

$+$

$\underline{\xi}$

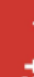

$\pm$

$\frac{9}{7}$

气

$\mathbb{1}$

চ

ISSN 2193-8806

\section{Haushalt in \\ Forschung}

\section{Qualifizierungsarbeiten Konsum - Ernährung - Gesundheit}
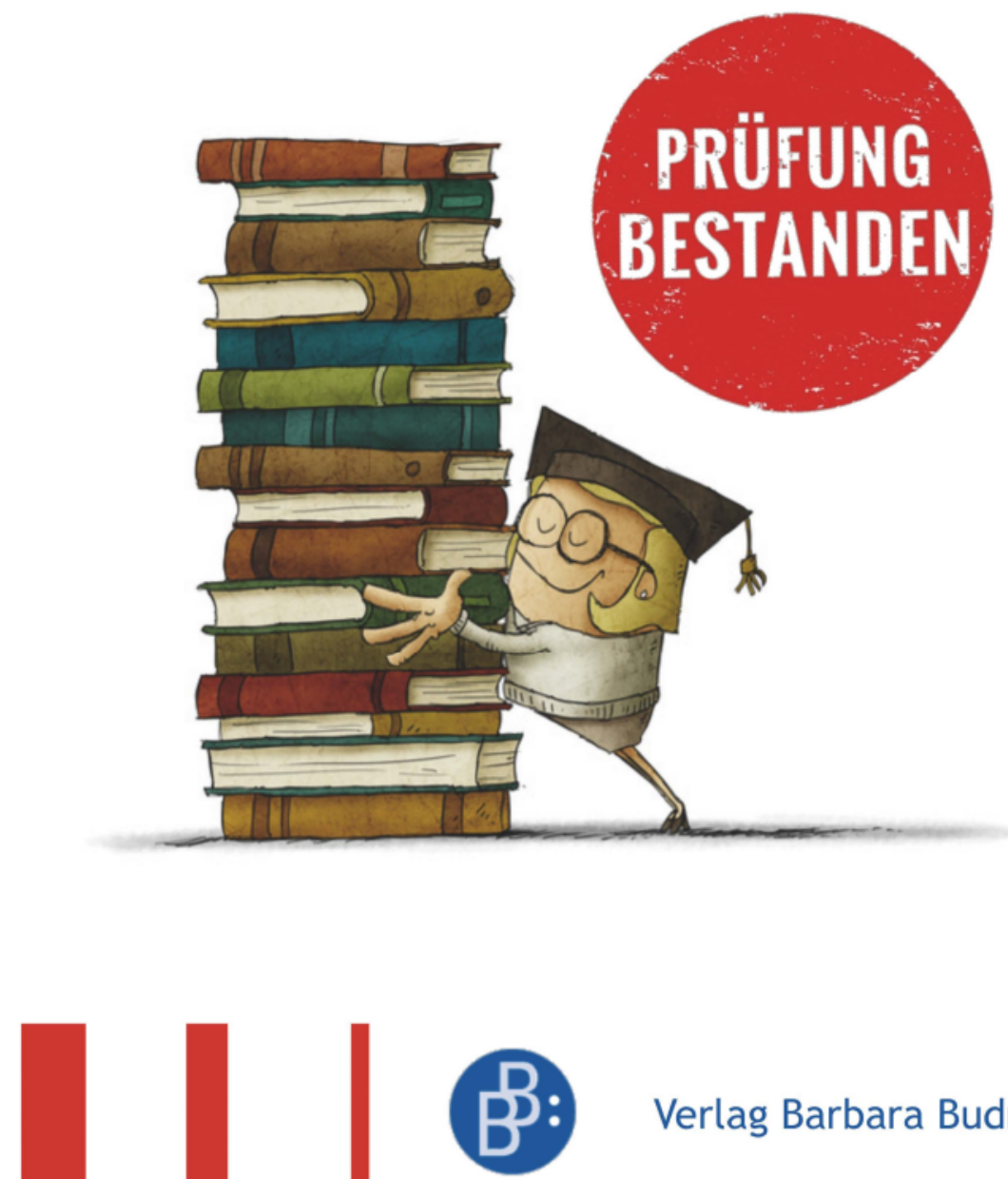

Verlag Barbara Budrich 
Inhaltsverzeichnis |

Werner Brandl

Editorial...... 2

Günther Koch

Wissenschaftliche Texte erschließen mit der Lesestrategie SQ3R 3

Martin Kornmeier

Kompetent wissenschaftlich schreiben

nach dem Gugelhupf-Prinzip.

Michael Booch

Zwischen Therapie und Beratung - Zur Rolle der Lehrkraft bei

der Präventions- und Interventionsarbeit von Essstörungen in der Schule.

Michaela Wölfer

Das Metabolische Syndrom im Jugendalter - Mögliche Ursachen

und Präventionsstrategien auf nationaler und internationaler Ebene

Julia Ernst

Aktuelle Herausforderungen bei der Schulverpflegung

an Gemeinschaftsschulen

Barbara Rieger

Essgenuss im Jugendalter.

Eva Steinfurth

Die Förderung des ethischen Ernährungsbewusstseins.

Andrea Balz \& Dina Limbach

Lernaufgaben und Unterrichtsmaterialien

zum neuen schweizerischen Lehrplan 21.

Birgit Happel

„Ich habe immer über meine Verhältnisse gelebt“ - Ein biografieanalytischer

Blick auf den Umgang mit Geld.

Tagungsankündigung

GFD Tagung 2017 Fachdidaktische Forschung zur Lehrerbildung 
| Essgenuss im Jugendalter

\section{Barbara Rieger}

\section{Essgenuss im Jugendalter}

(Ess-)Genusserlebnisse können mittel- und langfristig sowohl die körperliche als auch die psychische Gesundheit fördern. Darin liegt eine Chance, die bisher von der Ernährungs- und Verbraucherbildung kaum wahrgenommen wurde. Im Folgenden wird das Potenzial einer möglichen genussorientierten und genussfördernden Ernährungsbildung beleuchtet und zentrale Ergebnisse der zugrundeliegenden Masterarbeit vorgestellt, in der insbesondere hemmende und fördernde Faktoren für Essgenusserleben im Jugendalter identifiziert wurden.

Schlüsselwörter: Essgenuss, Schulverpflegung, EVB, Gesundheitsförderung

\section{Einleitung}

Bisherige Forschungen und Arbeiten zum Thema Genuss liegen vorwiegend aus dem Bereich der Psychologie (u. a. Lutz, 1983; Koppenhöfer, 2004, Bergler \& Hoff, 2002) vor. Innerhalb der Genussforschung wurden vor allem Genusstypen sowie die gesundheitlichen Auswirkungen von Genusserleben untersucht. Nur wenige Veröffentlichungen fokussieren speziell den Genuss im Zusammenhang mit Essen. Direkt oder indirekt beschäftigen sich beispielsweise Höhl (2009), Höhl \& Hahn (2013), Wierlacher (2008), Methfessel (2008; 2011), Methfessel, Höhn \& Miltner-Jürgensen (2016), Dürrschmid (2013), Barlösius (2011) und Schönberger (2005) mit der Thematik des Essgenusses. Im Bereich der EVB ist zudem vor allem Heindl (u. a. 2008) zu nennen, für die Essgenuss Teil ihres Konzeptes einer ästhetischen Bildung ist. Eine deutliche Forschungslücke zeigt sich hingegen bei der Zielgruppe der Jugendlichen. Deren Essgenussfähigkeit und -verständnis sowie förderliche Rahmenbedingungen wurden bisher kaum erhoben. Die dem hier vorliegenden Beitrag zugrundeliegende Masterarbeit „Essgenuss im Jugendalter“ (Rieger, 2016) soll eine erste Lücke schließen. Der Fokus liegt dabei auf der Betrachtung der hemmenden und fördernden Faktoren für das Essgenusserleben im Jugendalter. Darüber hinaus soll zudem aufgezeigt werden, wie eine stärkere Genussorientierung und -förderung einen Beitrag zu einer gelingenden Ernährungsund Verbraucherbildung leisten kann. 
Essgenuss im Jugendalter |

\section{Essgenuss - eine Begriffsbestimmung}

Um sich dem Thema Essgenuss zu nähern, muss vorab geklärt werden, welches Begriffsverständnis hier zugrunde gelegt wird. Die Genussforschung steht insgesamt noch relativ am Anfang und es zeigt sich insbesondere eine große Lücke bei der Zielgruppe der Jugendlichen. Darüber hinaus ergibt sich aus der aktuellen Forschungslage ein Mangel an wissenschaftlichen Genusstheorien und -definitionen (vgl. Bergler \& Hoff, 2002, S. 32). Keine der vorhandenen Definitionen beschreibt hinreichend, was hinter dem Wort Essgenuss zu verstehen ist. Erst eine Verbindung und Ergänzung verschiedener vorhandener Definitionsversuche zeichnet ein vollständiges Bild. Daher wird hier unter Rückgriff auf Formulierungen von Lutz (1983), Bergler und Hoff (2002), Höhl (2009) sowie von Wierlacher (2008) der Begriff Essgenuss wie folgt definiert und verwendet:

Essgenuss ...

- ist ein ganzheitliches, intensives, körperlich-sinnliches (Selbst-)Erleben, das emotional positiv besetzt ist.

- wird aktiv erlebt und herbeigeführt.

- ist zugleich bewusst und lustvoll sowie stets zeitlich begrenzt.

- ist an bestimmte individuelle Bedingungen geknüpft und schließt ein Geschmacksurteil mit ein.

- fördert Entspannung und bewirkt eine vielfältige Steigerung des körperlichen und seelischen Wohlbefindens und der Lebensqualität.

- ist eingebettet in eine Esssituation, in der sich das Genusserleben sowohl auf den eigentlichen Akt des Verzehrs, als auch auf das gesamte Ambiente und die stattfindende Kommunikation bezieht.

\section{Gesundheit und Genuss}

\subsection{Der Fehlschluss von Gesundheit und Genuss als Widerspruch}

Vielfach wird Genusserleben als Gefährdung für die Gesundheit dargestellt. Ein Beispiel hierfür findet sich selbst im aktuellen Bildungsplan 2016 BadenWürttemberg (Ministerium für Kultus, Jugend und Sport, 2016) für das Fach Alltagskultur, Ernährung und Soziales (AES). Dort steht, dass die Jugendlichen „subjektiv akzeptierte Wege zum Umgang mit möglichen Konflikten“" (ebd., S.17) herausarbeiten sollen. Als Beispiel wird der mögliche Konflikt zwischen Genuss und Gesundheit angeführt. In diesem Kontext scheint es, als würde sich ein gesundheitsförderlicher Lebensstil und Genusserleben gegenseitig ausschließen und Essgenuss eben gerade nicht zur Steigerung des körperlichen und seelischen Wohlbefindens 


\section{| Essgenuss im Jugendalter}

beitragen (vgl. Methfessel, 2013). Dazu passt auch, dass der Wert von Gesundheit seit einiger Zeit überall hoch im Kurs steht. Bei näherer Betrachtung fällt jedoch auf, dass Gesundheit oftmals zu einem ideologisch geprägten gesellschaftlichen Imperativ geworden ist (vgl. Klotter, 2010). Jeder ist demnach für seine persönliche Gesundheit selbst verantwortlich. Wer etwas auf sich hält, treibt möglichst viel Sport, orientiert sich an vorgeschriebenen Ernährungsempfehlungen und übt sich im Verzicht. Vorgegebene Normen unterscheiden dabei messerscharf zwischen ,richtigem“ und „falschem" Ernährungsverhalten (vgl. Reitermeier, 2013, S. 268f; Gruber, 2015, S. 35). Die psychische und soziale Bedeutung von Essen wird hier eher ausgeklammert. Aus dieser Perspektive scheint der Essgenuss der Gesundheit entgegen zu stehen. Gleichzeitig gilt jedoch, dass Menschen schon immer ein Bedürfnis nach Genusserleben hatten und haben (Bergler \& Hoff, 2002, S. 162).

Wie also ist der scheinbare Widerspruch aufzulösen? Dazu soll zunächst zwischen Genuss und Lust unterschieden werden. Genuss ist stets mit einem lustvollen emotionalen Zustand verbunden, schließt aber die Möglichkeit zur Selbststeuerung im Sinne von Selbstfürsorge mit ein und kann so auch einen Bedürfnisaufschub beinhalten. Selbstfürsorge meint hier, dass geregelt wird, „wann und wie genossen wird, wann es genug ist und wann und wie lange Askese sinnvoll und gut für den Einzelnen ist“ (Lutz \& Sundheim, 2002, S. 15). Dagegen ist Lust nicht immer genieBerisch und kann schlimmstenfalls zu süchtigem Verhalten führen (Lutz, 1983, S. 12f). Menschen mit ausgeprägter Genussfähigkeit verfügen darum in der Regel über eine flexible Verhaltenskontrolle. Sie treffen bewusste Entscheidungen, die einen selbstfürsorglichen Umgang ausdrücken und darum auch zu einer autonomen Lebensführung beitragen (Höhl, 2009, S 30; Lutz \& Sundheim, 2002, S.18f). Wer genießt und nicht allein seiner aktuellen Lust folgt, gibt sich also nicht einem hemmungslosen Schlemmen hin, sondern sucht und kreiert sich bewusste Genussmomente, die in einem ständigen Wechsel zur Askese stehen. Darum löst sich auch der scheinbare Widerspruch auf, denn "Gesundheit braucht Genuss“ (Höhl, 2009, S. 28), wie auch der folgende Abschnitt zeigen wird.

\subsection{Die positiven gesundheitlichen Auswirkungen von Genuss}

Positive Empfindungen nehmen Einfluss auf unser Wohlbefinden - und da (Ess-) Genusserleben stets mit positiven Emotionen verbunden ist, kann dieses im Sinne der Salutogenese dazu beitragen, Widerstandsressourcen zu stärken und das Wohlbefinden und die Gesunderhaltung zu fördern (vgl. Methfessel, 2008; Dr. Rainer WildStiftung, 2008, S. 2).

In verschiedenen Untersuchungen wurde konkret beleuchtet, welche positiven gesundheitlichen Auswirkungen Genusserleben mit sich bringt. Genuss übernimmt verschiedene intrapsychische Funktionen. Dazu gehören „die Kompensation des normalen Alltags, [...] die Entspannung, [sowie] die Belohnung [...] und die Anregung neuer psychischer Kräfte" (Bergler \& Hoff, 2002, S.40). Darüber hinaus konn- 


\section{Essgenuss im Jugendalter |}

ten Warburton und sein Team (2000) in ihrer Studie nachweisen, dass Genussmomente das Immunsystem stärken. Bereits genussvolle und damit freudige Erinnerungen erhöhen die Sekretion von Immunglobulin A (Antikörper gegen Viren) (a.a.O., S. 28). Vorfreude und Erinnerungen, die Teil des Genusses sind, nehmen darum aus gesundheitlicher Sicht eine wichtige Rolle ein. Insgesamt trägt Genusserleben dazu bei, den langfristigen Grundzustand der Lebenszufriedenheit zu verbessern. Im Gegensatz zu Genusszweiflern oder Genussunfähigen bewerten Genießer ihre Lebensqualität weit positiver (Bergler \& Hoff, 2002, S. 341f).

Bergler und Hoff (2002) zeigen in ihrer Studie zudem eindrücklich, dass gerade Genießende in der Regel einen sehr gesundheitsförderlichen Ernährungsstil pflegen. Sie stellen höhere Ansprüche an die Esssituation und nehmen sowohl den Geschmack als auch die Menge bewusster wahr (a.a.O., S. 306). Ein schönes Ambiente und eine anregende Kommunikation während des Essens sind für sie ein wichtiger Teil ihres Genussmomentes (ebd.). Zudem nehmen sich Genießer und Genießerinnen mehr Zeit zum Essen, was sich durch das verzögerte Einsetzen des Sättigungsgefühls positiv auf die Energiebilanz auswirkt. Im Vergleich zu Nichtgenießenden geben Genießende an, „deutlich mehr Obst und Gemüse zu konsumieren“ (Gruber, 2015, S. 74). Sie essen zudem insgesamt abwechslungsreicher. Genießer und Genießerinnen empfinden also eine gesundheitsförderliche Ernährungsweise als genussvoll. Dagegen empfinden Nichtgenießende eine gesundheitsförderliche Ernährungsweise als einen Widerspruch zum Verzehr von genussvollen Speisen.

Des Weiteren haben - entgegen gängigen Vorurteilen - Genießer und Genießerinnen in der Regel Normalgewicht und ein entspanntes Verhältnis zum Essen. So werden sie auch mit gesellschaftlichem und emotionalem Druck leichter fertig. Diäten kommen für sie kaum in Frage und sie neigen weder zum Frustessen, noch haben sie ein schlechtes Gewissen, wenn sie essen, was ihrem individuellen Geschmack entspricht (Bergler \& Hoff, 2002, S. 309). Da Über- und Untergewicht auch ein gesundheitsrelevantes Thema des Jugendalters darstellen (vgl. HBSC-Studienverbund Deutschland, 2015), kann die Ausbildung von Genussfähigkeit hier eine Unterstützung auf dem Weg hin zum Normalgewicht sein.

Die Ausführungen zeigen, dass Genuss zusammengefasst „Immunisierungs-, Ausgleichs- und Therapiefunktion" (Bergler \& Hoff, 2002, S. 169) besitzt. Damit kann eine verstärkte Genussorientierung eine entscheidende Größe für die Gesundheitsprävention und -förderung sein.

\section{Essgenuss(-fähigkeit) im Jugendalter}

Jugendlichen wird oftmals eine hedonistische Grundhaltung und damit auch eine eingeschränkte Genussfähigkeit unterstellt (Bergler \& Hoff, 2002, S. 266). Sie sind demnach kaum in der Lage Bedürfnisse aufzuschieben, sondern handeln vorwiegend lustbetont (Schulz, 2012, S. 4). Das Genusserleben Jugendlicher wird von Bergler 


\section{| Essgenuss im Jugendalter}

und Hoff (2002) als intensives Ausprobieren beschrieben, welches von dem Versuch-und-Irrtum-Prinzip geleitet ist und damit (noch) nicht mit einem umfassenden genussorientierten Lebensstil einhergeht (a.a.O., S. 267f). Gleichzeitig haben Jugendlichen ein starkes Genussbedürfnis und sehen Genuss als wichtigen Bestandteil ihres Lebens. Das Genussfeld des Essens spielt in dieser Altersgruppe durchaus eine Rolle (a.a.O., S. 71; 263). Die Entwicklung der Genussfähigkeit steht zudem im Zusammenhang mit den anstehenden Entwicklungsaufgaben. Auch hier werden Grenzen ausgetestet, eine soziale Verortung innerhalb von Peers und Familie findet statt, eigene Positionen müssen entwickelt werden und der Umgang mit dabei entstehenden Widersprüchen wird geschult (Bartsch, 2009, S. 92).

"Genussfähigkeit gehört zum reifen Erwachsenensein. Es ist eine gesellschaftliche Ressource und Kulturtechnik" (Gruber, 2015, S. 54), die zur Ernährungsbildung gehört (vgl. REVIS Rahmencurriculum der Ernährungs- und Verbraucherbildung der REVIS-Fachgruppe ${ }^{1}$; Schlegel-Matthies et al., in Vorbereitung). Die Ausbildung von Genussfähigkeit stellt demnach zweifellos einen Lern- und Entwicklungsprozess dar. Dadurch sind Unterschiede zwischen der Genussfähigkeit und dem Genusserleben zwischen Jugendlichen und Erwachsenen unleugbar vorgegeben. Dennoch wird hier die These vertreten, dass Jugendliche über ein weitreichenderes Genussverständnis verfügen, als ihnen weithin zugetraut wird. Dieser Annahme folgend, wurde in der durchgeführten Studie die Genussfähigkeit von Jugendlichen in den Blick genommen.

\section{Essgenuss in der Ernährungs- und Verbraucherbildung (EVB)}

Eine Ernährungs- und Verbraucherbildung, die sich vorwiegend an ernährungswissenschaftlichen Erkenntnissen orientiert und daraus rigide Ge- und Verbote ableitet, trägt meist wenig zu einer gesundheitsförderlichen Essweise bei (Reitmeier, 2013, S. 149; REVIS-Fachgruppe, 2005; Methfessel, 2005, S. 4ff.; Bartsch et al., 2013). „Es sind primär nicht kognitiv-rationale Überlegungen, die das menschliche Essverhalten steuern. Nicht Vernunft, sondern gelernte Bedürfnisse bestimmen die Speisenwahl, auch wenn ,kluge Argumente ' häufig als Gründe angeführt werden“ (Pudel, 2007, S. 308). Der informierte Mensch isst darum in der Regel dennoch in der gewohnten Art und Weise. Wenn er durch sein ernährungsbezogenes Wissen darüber hinaus das Gefühl erhält, sich falsch zu ernähren und das Essen mit einem schlechten Gewissen verbunden ist, dann kann dies negative Konsequenzen für dessen Wohlbefinden zur Folge haben (vgl. Dr. Rainer Wild-Stiftung, 2008, S. 3). Erst wenn der essende und handelnde Mensch in seiner jeweiligen Lebenswelt und mit seinen individuellen Bedürfnissen sowie die vielfältigen Funktionen, welche das Essen für ihn übernimmt, in den Fokus gerückt werden, kann die EVB langfristig ein selbstbestimmtes, gesundheitsförderliches Ess- und Ernährungsverhalten 


\section{Essgenuss im Jugendalter |}

fördern (Methfessel, 2005, S. 10ff; Methfessel, 2013; Schlegel-Matthies et al., in Vorbereitung). REVIS (Reform der Ernährungs- und Verbraucherbildung in Schulen) ist hier wegweisend (REVIS Fachgruppe, 2005). Essen bleibt als eine bedeutende Quelle für Genuss, Wohlbefinden und Lebensfreude weiterhin eher unterschätzt (Höhl, 2009, S. 34). Der (Bildungs-)Wert des Essgenusses für die EVB ist bisher vorwiegend unerforscht und ungenutzt. Ein Anliegen dieses Artikels ist es darum, die (Lern-)Chance, die in der großen Bedeutung von Genuss für Jugendliche liegt, aufzuzeigen.

\section{Essgenuss als Chance und Bildungsauftrag}

Essgenuss als Chance und Bildungsauftrag beinhaltet zweierlei. Auf der einen Seite tragen (Ess-)Genusserlebnisse zu einer Steigerung des Wohlbefindens bei und fördern somit mittel- und langfristig sowohl die körperliche als auch die psychische Gesundheit (siehe Absatz 2.2). Eine vermehrte Genussorientierung in allen Fächern und eine entsprechende Ausrichtung des Settings Schule können so Teil einer schulischen Gesundheitsförderung sein. Damit diese Form der Gesundheitsförderung erfolgreich sein kann, müssen verhaltens- und verhältnisbezogene Maßnahmen ineinandergreifen. Es müssen auf die Bedürfnisse der Jugendlichen abgestimmte Rahmenbedingungen geschaffen werden, damit Essgenusserlebnisse in der Schule für die Jugendlichen vermehrt möglich werden. Weiterhin sollte die Ausbildung von Genussfähigkeit bei den Jugendlichen konkret unterstützt werden.

Auf der anderen Seite stellt eine Genussorientierung in der Schule einen Ansatzpunkt dar, um das Lernen im Bereich Ernährung langfristig effektiver zu machen und über eine kognitive Verarbeitung hinaus auch die Handlungswirksamkeit zu fördern. Das Lernen über Essgenusserlebnisse beinhaltet, dass alle Sinnesorgane am Lernprozess beteiligt werden. Wissen wird also nicht mehr nur kognitiv angeeignet, sondern durch riechen, schmecken, sehen, hören und fühlen unterstützt. Die positiven Emotionen, die mit dem Essgenuss verbunden sind, tragen ebenfalls zum Lernerfolg bei. Diese Aussage lässt sich durch Ergebnisse der Hirnforschung untermauern. ${ }^{2}$ Ein genussorientierter Unterricht lässt sich jedoch ebenso mit dem Credo Pestalozzis begründen, welches Lernen „mit Kopf, Herz und Hand“ empfiehlt. Diese Form des Lernens ist vor allem deshalb so wünschenswert, weil „Geruch und Geschmack [...] vom ersten Lebenstag an mit wertenden Gefühlen verknüpft [sind], die Annahme und Ablehnung lebenslang begleiten" (Heindl, 2008, S.135). Durch biografische Erinnerungen an die sinnliche Wahrnehmung von Esssituationen und bestimmten Speisen und die dabei abgespeicherten Emotionen bildet sich im Laufe der Zeit ein emotionaler Index aus. Dieser nimmt starken Einfluss auf das Wohlbefinden und legt gleichzeitig fest, welche persönliche Bedeutung und welchen Wert eine Speise besitzt (vgl. ebd.). Genussorientiertes Lernen greift diese natürlichen Prozesse der Ernährungssozialisation auf. 


\section{| Essgenuss im Jugendalter}

Damit ein genussorientierter Unterricht erfolgsversprechend sein kann, muss zudem eine Ausrichtung an subjektorientiertem und salutogentisch orientiertem Lernen und Lehren vorgenommen werden (Bartsch, 2008b, S.100ff). Jeder Mensch benötigt andere Bedingungen zum Genießen, darum ist es unausweichlich den Einzelnen mit seinen individuellen Bedürfnissen und Motiven in den Blick zu nehmen. Essgenusserlebnisse können folglich eine Brücke schlagen zwischen dem Alltag der Jugendlichen und den fachlichen Inhalten und so auch der oftmals mangelnden Situiertheit im Unterricht begegnen (ebd., S.102). Durch einen genussorientierten Unterricht kann daher ein Beitrag zur Überbrückung der Diskrepanz zwischen Wissen und Handeln und damit zu einer erfolgreicheren Ernährungskommunikation geleistet werden.

\section{Methodisches Vorgehen}

\subsection{Fragestellung}

Der übergeordneten Forschungsfrage lag die zentrale Annahme zugrunde, dass für Jugendliche, ebenso wie für Erwachsene, bestimmte Einflussgrößen bestehen, die eine Voraussetzung für das Genusserleben darstellen und dieses sowohl fördern als auch verhindern können (vgl. Bergler \& Hoff, 2002, S.160). Aus dieser Annahme ergab sich die folgende übergeordnete Forschungsfrage:

„Welche Faktoren beeinflussen den Essgenuss im frühen Jugendalter?"

Die Beantwortung der Frage setzte eine Beschäftigung mit dem subjektiven Genussverständnis der Jugendlichen voraus, denn je nach dem, was subjektiv als Essgenussmoment bestimmt und wahrgenommen wird, leiten sich daraus Bedingungen ab, die ein solches Essgenusserleben erst ermöglichen oder im umgekehrten Fall verhindern. Von weiterem Interesse war die Frage nach der allgemeinen Genussfähigkeit von Jugendlichen. Die Beantwortung ist von besonderer Relevanz, da den Jugendlichen in der Literatur oftmals eine vorherrschende Lustorientierung unterstellt wird (vgl. Schulz, 2012, S. 4). Darüber hinaus wurde eine erste Exploration zur Frage durchgeführt, worin sich die Jugendlichen von den Erwachsenen hinsichtlich ihres Genusserlebens unterscheiden.

\subsection{Datenerhebung und Auswertung}

Stichprobe: Die Stichprobe setzte sich aus zehn Jugendlichen $(\mathrm{N}=10)$ im Alter von 13 und 17 Jahren zusammen. Die Erhebung bezog sich damit explizit auf das frühe Jugendalter (vgl. Hurrelmann, 2007, S. 41) und wurde von den (Alters-)Rändern der frühen Jugendphase her erschlossen, um so ein Verständnis für die Entwicklung in dieser Phase zu erwerben (vgl. Flick, 2007, S. 165). Es wurden vier Mädchen und 
Essgenuss im Jugendalter |

sechs Jungen befragt, die Realschulen oder Gymnasien besuchten. Jugendliche anderer Schulformen waren nicht beteiligt.

Forschungsmethode: Zur Erhebung der Daten wurde die Methode des qualitativen Leitfadeninterviews verwendet. In den Leitfaden wurden als wichtig erachtete Aspekte aus der bisherigen Genussforschung und -literatur eingeflochten, um das Prinzip des theoriegeleiteten Vorgehens zu realisieren. Der qualitative Zugang ermöglichte den Jugendlichen als Expertinnen und Experten ihrer Lebenswelt von ihren Genusserfahrungen und -vorstellungen zu berichten. Die Erstellung des Leitfadens erfolgte in Anlehnung an die Empfehlungen von Gläser und Laudel (2009, S. $111 \mathrm{ff}$.).

Transkription und Auswertung: Die Interviews wurden für die Analyse und Interpretation digital aufgezeichnet. Für die Verschriftung wurden die Konventionen der Transkription nach Flick (2007) verwendet und teilweise durch Transkriptionsregeln von Kuckartz (2014) ergänzt (vgl. S. 136f). Ausgewertet wurde das Datenmaterial mithilfe des systematischen und regelgeleiteten Verfahrens der qualitativen Inhaltsanalyse nach Kuckartz (2014). Aufgrund der Arbeit mit thematischen Kategorien wurde auf die Basismethode der inhaltlich strukturierenden Inhaltsanalyse zurückgegriffen (vgl. a.a.O., S. 75ff). Die Kategorien wurden dabei in einem mehrstufigen Verfahren sowohl deduktiv als auch induktiv gewonnen. Zur einfacheren Datenorganisation fand die Auswertung computerunterstützt mithilfe der MAXQDA-Software statt (vgl. a.a.O., S. 133ff).

\section{Jugendlicher Essgenuss}

\subsection{Ergebnisse und Interpretation zu den hemmenden und fördernden Einflussfaktoren auf den Essgenuss von Jugendlichen}

Die Faktoren, die sich auf den Essgenuss im Jugendalter beziehen, können sowohl positiv als auch negativ wirken. Aufgrund dessen wurden die beiden Hauptkategorien "Genussförderer" und „Genussverhinderer" gebildet. Alle Subkategorien wurden wiederum verschiedenen inhaltlichen Themenbereichen zugeordnet. Die Themenbereiche umfassen Faktoren, die sich auf das Essen selbst, die Gesellschaft, die Atmosphäre, die Zeit oder auf den Ort beziehen. Für Faktoren, die sich diesen Kategorien nicht zuordnen ließen, wurde die Kategorie Sonstiges eingeführt. ${ }^{3}$

Genussförderer und Genussverhinderer bezogen auf das Essen: Unabhängig von Alter und Geschlecht spielt der Geschmack für alle eine zentrale Rolle im $\mathrm{Zu}$ sammenhang mit Essgenusserleben. Damit Essgenuss möglich wird, muss das Essen für die Jugendlichen „schmackhaft" sein. Ein als mangelhaft empfundener Geschmack (,,nicht schmackhaft") wird dagegen von den meisten als Hinderungsgrund genannt. Das Ergebnis ist wenig überraschend, es bestätigt die hohe Relevanz des 


\section{| Essgenuss im Jugendalter}

Geschmacks, die auch andere Studien für alle Altersgruppen (z. B. Pudel, 2007, S. $308 \mathrm{ff}$.) und explizit für Jugendliche (Bartsch, 2008a) bereits belegt haben. Überraschend dagegen ist, dass die Jugendlichen (gerade auch die Mädchen) neben Spaghetti, Pizza, Kuchen und Co. besonders gerne Fleisch genießen.

Zum Essgenuss trägt für die Jugendlichen zudem durchgängig bei, wenn das Essen ,selbstgemacht" ist (vgl. dazu auch Dohle, Rall \& Siegrist, 2016). Selbstgemacht bezieht sich an dieser Stelle nicht nur auf Gerichte, die die Jugendlichen selbst zubereitet haben, sondern vorwiegend auf solche Speisen, die von anderen Familienmitgliedern, insbesondere von der Mutter oder der Großmutter oder in gemeinsamer Arbeit hergestellt wurden. Durch selbstgemachtes Essen und den damit verbundenen Aufwand fühlen die Befragten sich wertgeschätzt. Das selbstgemachte Essen verbinden sie mit Aufmerksamkeit und Zuwendung, weshalb es in Bezug auf den Essgenuss von großer Bedeutung ist. Positive Gefühle werden so unterstützt und der Essgenuss gefördert. Diese selbstgemachten Lieblingsgerichte sind oftmals mit positiven Erinnerungen verknüpft und haben sich durch die Ernährungssozialisation in der Familie zur Vorliebe entwickelt. Hier zeigt sich deutlich, wie wichtig die emotionale Komponente für den Essgenuss ist und wie stark Vorfreude und vergangene Erfahrungen von Bedeutung für das Essgenusserleben sind. Haben die Jugendlichen das Essen selbst zubereitet, dann spielt zudem der Stolz auf die eigene Leistung eine Rolle und steigert den Genuss beim Essen. „Fertigprodukte "verhindern dagegen für sieben der Befragten Genusserlebnisse. Beispielsweise genießt Matthias (13 Jahre) vor allem, wenn es ,öfters mal so was wirklich leckeres Selbstgemachtes hat und manchmal nicht einfach aus der Tüte" kommt. Dahinter stecken verschiedene Beweggründe. Zum einen gilt Fertigessen im Gegensatz zu Selbstgemachtem als lieblos. Darüber hinaus verbinden einige der Jugendlichen damit auch negative Gefühle, da sie die Inhaltsstoffe als gesundheitsschädlich einschätzen.

Ist das Essen ,,schön angerichtet “ fördert dies für insgesamt sieben der Jugendlichen den Genuss beim Essen. Wohingegen ,lieblos angerichtete" Speisen von drei der 17-jähigen als genussverhindernd angeführt werden. Die Art und Weise der Lebensmittelanrichtung ist demnach ebenfalls ein wichtiger Faktor, der das Essgenusserleben fördern oder hemmen kann. Hier spiegeln sich die Ergebnisse von Jansen et al. wieder. Diese haben in ihrer Erhebung nachgewiesen, dass eine attraktive Produktpräsentation der Speisen in der Schulmensa erheblichen Einfluss auf das Kaufverhalten der Jugendlichen nimmt (vgl. Jansen et al., 2012, S. 188).

Den Aufwand der hinter der Zubereitung der Speisen steckt, thematisieren lediglich die älteren Befragten. „Aufwendig zubereitete“ Gerichte erhöhen den Genusswert für zwei der Befragten, wohingegen drei der Siebzehnjährigen einen allzu großen Aufwand (, zu aufwendig ") eher als genussverhindernd ansehen. Hier deutet sich auch ein Zusammenhang zwischen den vorhandenen küchentechnischen Fertigkeiten und der Bereitschaft aufwendigere Speisen selbst zuzubereiten an. Je ausgeprägter 


\section{Essgenuss im Jugendalter |}

die vorhandenen Kompetenzen sind, desto eher wird die Zubereitung selbst Teil des Genusses.

Vereinzelt wurde zudem genannt, dass am liebsten etwas als ,,gesund“ empfundenes genossen wird. Etwas „,zu Fettiges“ lässt dagegen für drei der Befragten keinen Essgenuss zu. Auffällig ist hier die (subjektive) Bedeutung von Gesundheit bereits im Jugendalter. So wurde der Zusammenhang zwischen Gesundheit und dem Essverhalten in jedem der geführten Interviews direkt oder indirekt thematisiert. „Gesundheit" wird von den Jugendlichen durchaus ernst genommen. Es ist davon auszugehen, dass sich die alterstypischen subjektiven Bedeutungen von "Gesundheit“ von anderen Altersgruppen unterscheiden (vgl. z. B. Bartsch, 2008a), das war jedoch nicht Gegenstand dieser Studie. Die Ergebnisse von Jansen et al. (2012, S.187) weisen ebenfalls auf die Bedeutung der Gesundheit im Jugendalter hin. Das deutet darauf hin, dass der Gesundheitstrend und der damit verbundene gesellschaftliche Druck auch bei den Jugendlichen schon zu spüren ist. Zum anderen widersprechen sich die Jugendlichen hier häufiger, indem sie an anderer Stelle thematisieren, wie gerne sie Kuchen oder Süßes genießen (was für sie selbst in die Kategorie „ungesund" fällt). Dies kann als Zeichen gedeutet werden, dass das jugendliche Essverhalten oftmals noch nicht vollständig gefestigt ist. Diese Formbarkeit und Offenheit kann und sollte von der EVB als Chance erkannt und genutzt werden. Gleichzeitig zeigen die scheinbaren Widersprüchlichkeiten aber auch lediglich die situative Abhängigkeit von Essgenusserleben. Je nach Situation wird (auch von Erwachsenen) unterschiedlich gewertet und genossen.

Aspekte der Nachhaltigkeit spielen für die Jugendlichen dagegen eher eine untergeordnete Rolle im Zusammenhang mit Essgenuss oder Essen im Allgemeinen. Nur einer der Befragten 17-Jährigen genießt sein Essen mehr, wenn es „,nachhaltig produziert" wurde. Die "mangelnde Qualität der Lebensmittel" nennen zwei der Befragten als genusshemmend. „Unhygienische“ Zustände verhindern für fünf der Jugendlichen Essgenussmomente. Auffällig ist hier vor allem, dass die Aussagen ausschließlich im Zusammenhang mit der eigenen Schulmensa gefallen sind. Generell wurde die Versorgung in der Schule durchweg negativ bewertet, sowohl bezogen auf das Essen selbst, als auch auf die Atmosphäre und die räumliche Gestaltung. Hier zeigt sich ein deutlicher Handlungsbedarf.

Genussförderer und Genussverhinderer bezogen auf die Gesellschaft: Mit Ausnahme zweier 17-jähriger Jungen (einer genießt am liebsten ,,alleine “, der anderer hat hier , keine Präferenzen") genießen Jugendliche besonders häufig in Gesellschaft. Damit ist die Gesellschaft in der Esssituation ein wichtiger genussfördernder Faktor. Besonders gerne wird in der Gemeinschaft ",mit der Familie“, ,mit Freunden " oder "mit der Oma und/oder dem Opa" genossen. Die Familie besitzt für die Jugendlichen im Zusammenhang mit Essgenuss einen besonders hohen Stellenwert. Diese Wertschätzung der Familie entspricht den Ergebnissen der Shell-Jugendstudie (2015). Die Koppelung von Essen mit einem Gefühl der Sicherheit und des Ver- 


\section{| Essgenuss im Jugendalter}

sorgtseins durch die Familie könnte zu diesem Ergebnis beitragen. Darüber hinaus müssen die Jugendlichen hier niemandem etwas beweisen, was eine entspannte und genussfördernde Haltung zulässt. Im Zusammenhang mit Freunden wird Essen dagegen teilweise zur Nebensache, weil eher das gemeinsame Erleben im Mittelpunkt steht (vgl. Bartsch, 2008a, S. 81; Reitmeier, 2013, S.179).

Mit zwei Ausnahmen sind „Gespräche beim Essen “ für alle ein wichtiger Teil des Essgenusses. „Keine guten Gespräche" verhindern dagegen Essgenuss. Viele Interviewausschnitte lassen gar den Schluss zu, dass die Gesellschaft und die Unterhaltung für das Genusserleben entscheidender sind, als das Essen selbst. Die Gemeinschaft steht im Vordergrund und das Essen wird gerade auch dann positive bewertet und genossen, wenn es "alle zusammenbringt". Dazu passt auch, dass "gemeinsames Zubereiten" von Speisen für vier der Befragten ein Bestandteil des Genusserlebnisses sein kann. Die Ergebnisse belegen, wie groß der Einfluss emotionaler und sozialer Aspekte auf den Essgenuss ist.

Genussförderer und Genussverhinderer bezogen auf die Atmosphäre: Bezogen auf die Atmosphäre wurden folgende Faktoren als genussförderlich genannt (mit unterschiedlicher Häufigkeit). Eine „schönes Ambiente“, „viel Platz", eine "gemütliche" und "dekorierte" sowie ,ruhige " Umgebung in der es ,unförmlich" zugeht, unterstützt für die befragten Jugendlichen tendenziell Essgenusserleben. Gegenteilig wirkt sich hingegen für die Befragten eine "schlechte Raumgestaltung ", eine ",unruhige“, „zu laute ", "unordentlich" Umgebung oder ,keinen Platz" zu haben aus und sie empfinden es als unangenehm, wenn ,zu viel nebenher passiert" oder die Atmosphäre zu ,förmlich" ist. Die Genussregel „Genuss geht nicht nebenbei“" (Koppenhöfer, 2004, S.25) hat der Großteil der Jugendlichen demnach schon für sich entdeckt. Auch Jansen et al. (2012) generieren ähnliche Ergebnisse im Zusammenhang mit der Schulverpflegung, wonach Jugendliche großen Wert auf die räumliche Gestaltung legen und dort keinen Lärm möchten (vgl. ebd. S. 187).

Darüber hinaus wird von acht der Befragten eine „positive Grundstimmung " als Voraussetzung für Essgenusserleben genannt. Dagegen kann eine ,negative Grundstimmung", die damit zusammenhängen kann, dass die Jugendlichen sich ,gestresst" oder "unwohl fühlen" Genussmomente vereiteln. Die Grundgestimmtheit der Jugendlichen ist demnach ebenfalls bedeutsam für die Wahrnehmung von Essgenussmomenten.

Genussförderer und Genussverhinderer bezogen auf Zeit und Ort: Fünf der Jugendlichen (drei davon 13 Jahre) bevorzugen den „häuslichen " Genuss. Vier der Befragten genießen hingegen sowohl Zuhause als auch ,außerhäuslich“. Lediglich einer der Befragten genießt generell lieber außer Haus. Das bevorzugt häusliche Genießen der 13-Jährigen könnte mit deren eingeschränkter Mobilität zusammenhängen. Zudem ist der Ablösungsprozess und der Drang Neues auszuprobieren vermutlich noch nicht so weit fortgeschritten. 


\section{Essgenuss im Jugendalter |}

Bezogen auf die Zeit gibt es zwei zentrale Ergebnisse. Zum einen ist es für die Jugendlichen ein wichtiges Kriterium, dass sie „Zeit haben" oder sich diese bewusst nehmen (für 9 von 10 Befragten). Zum anderen stört ,keine Zeit zu haben " das Genusserleben. Die Jugendlichen scheinen sich also schon bewusst Freiräume für Genussmomente schaffen zu können und sind sich der Genussregel „Genuss braucht Zeit" (Koppenhöfer, 2004, S.26) bewusst. Einig sind sich zudem alle Befragten darin, dass ein "außergewöhnlicher Anlass" besonders zum Genießen anregt. Hier zeigt sich besonders bei den älteren Befragten der Wunsch nach etwas Außergewöhnlichem und die entwicklungsbedingte Lust auf Neues (vgl. Schulz, 2012, S.4). Im Alltäglichen Genussmomente zu entdecken und zu kreieren fällt den Jugendlichen demnach hingegen noch schwer.

\subsection{Beurteilung der Genussfähigkeit der Jugendlichen und Bestimmung des jugendspezifischen Genusses}

Die Genussfähigkeit der Jugendlichen ist individuell sehr verschieden, wie es auch bei Erwachsenen der Fall ist. Es lassen sich lediglich einige Tendenzen aufzeigen. So ist die Fähigkeit Genuss im Alltäglichen zu suchen, tendenziell noch wenig ausgeprägt. Darüber hinaus ist das Genussspektrum der Jugendlichen in der Regel noch relativ klein und beschränkt sich auf bestimmte Speisen (bevorzugt werden Süßspeisen, Pizza und Pasta sowie Fleischgerichte). Dennoch zeigte die Auswertung der Interviews eine Entwicklung der Genussfähigkeit zwischen dem 13. und 17. Lebensjahr. Mit zunehmendem Alter tritt die Fähigkeit zum Maßhalten vermehrt hervor und auch die jeweiligen Präkonzepte von Genuss werden komplexer. Pauschalisierende Aussagen über die mangelnde Genussfähigkeit von Jugendlichen können insgesamt also zurückgewiesen werden. Als jugendspezifisch hat sich die Suche nach außergewöhnlichen Anlässen für Essgenusserlebnisse herauskristallisiert.

\section{Folgerungen für die Ernährungsbildung}

Folgerungen für die Schulverpflegung: In den Interviews wurde deutlich, dass die Schulverpflegung für die Jugendlichen häufig im Zusammenhang mit genussverhindernden Faktoren steht. Die Ergebnisse lassen den Schluss zu, dass viele Schulen ihrer Verantwortung als Ernährungssozialisationsinstanz wenig gerecht werden und alles andere als genussförderlich gestaltet sind. Durch ein abwechslungsreiches und genussförderliches Schulverpflegungsangebot kann das Essverhalten Jugendlicher jedoch nachhaltig beeinflusst und die Gesundheit gefördert werden.

Produktangeboten sollten darum zukünftig , über die reine Funktionalität des ,SattMachens' hinausgehen, sie müssen Bedürfnisse auf der kognitiven, emotionalen und sozialen Ebene erfüllen, das heißt, sie müssen positive Gefühle erzeugen" (Jansen et al., 2012, S. 188). Schulverpflegungsangebote müssen sich an den Bedürfnissen der 


\section{| Essgenuss im Jugendalter}

Jugendlichen ausrichten, um überhaupt attraktiv zu sein. Damit Genusserleben möglich und eine Geschmackserweiterung angebahnt werden kann, sollte ein breites und vielfältiges Angebot geschaffen werden, das sich am Geschmack der Jugendlichen ausrichtet und sie erst langsam an neue Geschmäcker gewöhnt. Bezogen auf die vorgestellten Ergebnisse sollte das Schulessen am besten frisch und vor Ort zubereitet sein und liebevoll angerichtet und präsentiert werden (vgl. hierzu auch Jansen et al., 2012, S. 188). Zudem sollte ein jugendgerechtes Ambiente und ein ausreichendes Zeitfenster für das Essen eingeplant werden. Des Weiteren sollte eine lockere und entspannte Atmosphäre, in der Gespräche ihren festen Platz erhalten, angestrebt werden. Wünschenswert wäre es darüber hinaus eine Beteiligungskultur zu schaffen, in der die Jugendlichen selbst ihre Wünsche äußern können, um so auch ihrem Bedürfnis nach Mit- und Selbstbestimmung nach zu kommen.

Folgerungen für den Fachunterricht: Da laut den Ergebnissen Esssituationen jugendspezifisch vor allem zu außergewöhnlichen Anlässen genossen werden, ergeben sich daraus vielfältige Möglichkeiten für den Unterricht. Um Essgenussmomente zu ermöglichen, könnten im Unterricht besondere Anlässe und Erlebnisse kreiert werden. Das bedeutet nicht, dass es besonders aufwendige Essensinszenierungen sein müssen; hier geht es vielmehr darum Einzelaspekte herauszuarbeiten und gemäß der Begriffsverwendung (Abschnitt 1) emotional positive Erlebnisse zu schaffen, die u.a. aktiv in entspannter und angenehmer Situation mit allen Sinnen erlebt werden können. Damit können Jugendliche dazu angeregt werden, auch in alltäglichen Kleinigkeiten Genussmöglichkeiten zu erkennen.

Sinnesschulungen sollten eine differenziertere Wahrnehmungsfähigkeit systematisch entwickeln und damit die Geschmackswahrnehmung differenzieren und eine Geschmackserweiterung unterstützen. Zudem scheint es bedeutsam, im Unterricht Genussverbote und -tabus explizit zu thematisieren, um einen schuldgefühlfreien Umhang mit Essen zu ermöglichen. Hier könnten biografische Methoden hilfreich sein. Der Faktor, dass die Jugendlichen vor allem in Gemeinschaft Genuss erleben, muss ebenfalls im Unterricht berücksichtigt werden. Gemeinsames Zubereiten und Erleben fördert den Essgenuss.

Generell zeigen die Ergebnisse, dass das Genussbedürfnis der Jugendlichen in der Regel relativ groß ist und die Fähigkeiten zum Genießen zwar vorhanden, aber noch nicht alle vollständig ausgebildet sind. Die EVB sollte die Ausbildung der Genussfähigkeit der Schülerinnen und Schüler darum weiter fördern.

\section{Ausblick}

Die durchgeführte, explorative Studie ist durch die Stichprobe in Umfang und Auswahl der Interviewpersonen limitiert. Wünschenswert wäre eine vertiefende Studie mit mehr Jugendlichen aus verschiedenen Milieus. Spannend wäre u. a., ob eine stärkere Lustorientierung vom sozialen Milieu abhängig ist. Darüber hinaus wäre die 


\section{Essgenuss im Jugendalter |}

Erarbeitung eines differenzierten Leitfadens und Tests zur genaueren Erfassung der Genussfähigkeit der Jugendlichen interessant. Wünschenswert im Sinne der Methodentriangulation wäre zudem die Ergänzung durch eine großflächige quantitative Studie. Die vorliegenden Ergebnisse legen nahe, das Setting Schule und den Unterricht genussförderlicher zu gestalten. Damit könnte ein Beitrag für die Gesundheitsförderung geleistet werden. Darüber hinaus sollte die Ernährungsbildung Essgenuss als Chance sehen und nutzen, um Lernen mit positiven Emotionen in Verbindung zu bringen und so eine Geschmacksvielfalt zu akzeptieren.

Die Genussfähigkeit der Schülerinnen und Schüler ist im Sinne von REVIS ein Beitrag zur Allgemeinbildung (vgl. auch Schlegel-Matthies et al., in Vorbereitung) und sollte daher gezielt angebahnt werden. Sinnesschulungen und Erlebnisinszenierungen sind nur Beispiele für eine Vielzahl von Möglichkeiten. Die EVB benötigt hier ausgereifte Konzepte zur Anbahnung von Genussfähigkeit, welche bisher noch weitgehend ausstehen.

\section{Danksagung}

Ein herzliches Dankeschön für die Betreuung der Masterarbeit und die Unterstützung bei der Umsetzung dieses Artikels geht an Frau Prof. Dr. Silke Bartsch.

\section{Anmerkungen}

1 Im Kooperationsprojekt „Reform der Ernährungs- und Verbraucherbildung in Schulen" (REVIS) wurde in den Jahren 2003 bis 2005 ein Curriculum für die Ernährungs- und Verbraucherbildung entwickelt: Detaillierte Informationen finden sich unter http://www.evb-online.de/evb_revis.php; in Fortsetzung dazu auch Schlegel-Matthies et al., (in Vorbereitung).

2 Der emotionale Zustand beim Lernen ist ausschlaggebend dafür, wo (im Hippocampus oder im Mandelkern) neue Informationen im Gehirn gespeichert werden. In einem emotional positiv besetzten Kontext werden Inhalte mit persönlicher Bedeutsamkeit im Hippocampus gespeichert. Dort werden die Einzelheiten nicht nur abgelegt, sondern so transformiert, sodass sie über die Gehirnrinde langfristig zur Verfügung stehen und zur Problemlösung genutzt werden können (vgl. Spitzer, 2006, S. 28f).

3 Die Zuordnung soll für eine größere Übersichtlichkeit sorgen. Es wären aber durchaus auch andere Möglichkeiten der Darstellung und Unterteilung möglich gewesen. 


\section{| Essgenuss im Jugendalter}

\section{Literatur}

Barlösius, E. (2011). Soziologie des Essens. Eine sozial- und kulturwissenschaftliche Einführung in die Ernährungsforschung (2. Aufl.). Weinheim: Juventa.

Bartsch, S. (2008a). Jugendesskultur. Bedeutungen des Essens für Jugendliche im Kontext Familie und Peergroup. Köln: BZgA .

Bartsch, S. (2008b). Subjektorientierung in der Ernährungs- und Gesundheitsbildung. Ernährung - Wissenschaft und Praxis, 2(3), 100-106.

Bartsch, S. (2009). Einflüsse auf das Essverhalten Jugendlicher. In M. Kersting (Hrsg.), Kinderernährung aktuell. Schwerpunkte für Gesundheitsförderung und Prävention (S. 92-101). Sulzbach: Umschau Zeitschriften Verlag.

Bartsch, S., Büning-Fesel, M., Cremer, M., Heindl, I., Lambeck, A., Lührmann, P., Oepping, A., Rademacher, C. \& Schulz-Greve, S. für die DGE-Fachgruppe Ernährungsbildung (2013). Spezial Ernährungsbildung. ErnährungsUmschau, 60(2) M84-M95.

Bergler, R. \& Hoff, T. (2002). Genuss und Gesundheit. Köln: KUV.

Deutsche Shell Holding GmbH (2015). 17. Shell Jugendstudie: eine pragmatische Generation im Umbruch. http://www.shell.de/aboutshell/media-centre/news-andmedia-releases $/ 2015 /$ shell-jugendstudie.html

Dohle, S., Rall, S. \& Siegrist, M. (2016). Does Self-Prepared Food Taste Better? Effects of Food Preparation on Liking. Health Psychology, 35(5), 500-508.

Dr. Rainer Wild-Stiftung (Hrsg.). (2008). Wie viel Genuss tut gut? (Themenpapier Dr. Rainer Wild-Stiftung, Ausgabe 1, Fakten, Trends und Meinungen. Gesunde Ernährung interdisziplinär aufbereitet).

http://www.gesunde-ernaehrung.org/images/Dr_Rainer_Wild_Stiftung/ 07_Presse/Themenpapier/pdf/Wie_viel_Genuss_tut_gut.pdf

Dürrschmid, K. (2013). Vom Glück des Schmeckens. Psychologische Aspekte des Schmeckens. In Dr. Rainer Wild-Stiftung (Hrsg.), Käsebrot mit Marmelade (S. 115-137). Heidelberg.

Flick, U. (2007). Qualitative Forschung. Eine Einführung (7. Aufl.). Reinbek bei Hamburg: Rowohlt.

Gläser, J. \& Laudel, G. (2009). Experteninterviews und qualitative Inhaltsanalyse. Als Instrumente rekonstruierender Untersuchungen (3. Aufl.). Wiesbaden: VS Verlag für Sozialwissenschaften.

Gruber, M. (2015). Mut zum Genuss. Warum uns das gute Leben gesund und glücklich macht. Wien: edition a.

HBSC-Studienverbund Deutschland (2015). Faktenblatt zur Studie Health Behaviour in School-aged Children 2013/14.

http://www.gbe-bund.de/pdf/Faktenbl_Koerpergewicht_2013_14.pdf

Heindl, I. (2008). Kulinaristik und Allgemeinbildung. In A. Wierlacher \& R. Bendix (Hrsg.), Kulinaristik. Forschung-Lehre-Praxis (S. 129-146). Berlin: Lit. 


\section{Essgenuss im Jugendalter |}

Höhl, K. (2009). Gesundheit braucht Genuss. Haushalt \& Bildung, 86(1), 28-35.

Höhl, K. \& Hahn, L. (2013). Essen wir Dinge, die uns nicht schmecken? In Dr. Rainer Wild-Stiftung (Hrsg.). Käsebrot mit Marmelade (S. 103-114). Heidelberg.

Hurrelmann, K. (2007). Lebensphase Jugend. Eine Einführung in die sozialwissenschaftliche Jugendforschung (9. Aufl.). Weinheim: Juventa Verlag.

Jansen, C., Schreiner-Koscielny, J., Eilenberger, S. \& Koscielny, G. (2012): Die komplexe Realität der Schulverpflegung. Teil 2: Wünsche und Wirklichkeit was Schüler (zum Essen) bewegt. Ernährung im Fokus, 12(05-06),186-189.

Klotter, C. (2010). Gesundheit als Pflicht? Das Dilemma der Gesundheitsförderung. Ernährung im Fokus, 10(5), 190-195.

Koppenhöfer, E. (2004). Kleine Schule des Genießens. Ein verhaltenstherapeutisch orientierter Behandlungsansatz zum Aufbau positiven Erlebens und Handelns. Lengerich: Pabst Science Publisher.

Kuckartz, U. (2014). Qualitative Inhaltsanalyse. Methoden, Praxis, Computerunterstïtzung (2. Aufl.). Weinheim: Beltz Juventa.

Lutz, R. (1983). Genuß und Genießen. In R. Lutz (Hrsg.), Genuß und Genießen. Zur Psychologie des genußvollen Erlebens und Handelns (S. 11-18). Weinheim: Beltz.

Lutz, R. \& Sundheim, D. (2002). Das Euthyme Konzept: Genuss zum Wohle der Gesundheit. Psychologische Aspekte gesundheitsfördernder Ernährung. Internationaler Arbeitskreis für Kulturforschung des Essens, 9, 14-24.

Methfessel, B. (2005). REVIS Fachwissenschaftliche Konzeptionen: Soziokulturelle Grundlagen der Ernährungsbildung. Paderborn: Universität Paderborn.

Methfessel, B. (2008). Salutogenese - ein Modell fordert zum Umdenken heraus Teil 2. Herausforderungen für die Gesundheitsförderung. ErnährungsUmschau, 55(1), 37-43.

Methfessel, B. (2013). Essen genießen als Beitrag zu Lebensqualität und Wohlbefinden. ,Die Säule“, 1, 30-33.

Methfessel, B., Höhn, K. \& Miltner-Jürgensen, B. (2016). Essen und Ernährungsbildung in der KiTa. Entwicklung - Versorgung - Bildung. Stuttgart: Kohlhammer.

Ministerium für Kultus, Jugend und Sport Baden-Württemberg (Hrsg.). (2016). Gemeinsamer Bildungsplan der Sekundarstufe I. Alltagskultur, Ernährung, Soziales (AES). Stuttgart.

Pudel, V. (2005). Sicherheit und Lebensqualität durch sensorische Lust. In D. von Engelhardt, R. Wild \& G. Neumann (Hrsg.), Geschmackskulturen. Vom Dialog der Sinne beim Essen und Trinken (S. 59-70). Frankfurt a. M.: Campus.

Pudel, V. (2007). Was Menschen motiviert richtig zu essen. Wie verbessert Ernährungsberatung ihren Erfolg? Teil 1: Prinzipien der Ernährungsberatung. Ernährungs Umschau, 54(6), 308-313.

Reitmeier, S. (2013). Warum wir mögen, was wir essen. Eine Studie zur Sozialisation der Ernährung. Bielefeld: transcript. 


\section{| Essgenuss im Jugendalter}

Rieger, B. (2016). Essgenuss im Jugendalter. Masterarbeit an der Pädagogischen Hochschule Karlsruhe, Studiengang: Master Bildungswissenschaften, Schwerpunkt Alltagskultur und Gesundheit. Abgabe am 31.05.2016.

REVIS-Fachgruppe (2005). REVIS Schlussbericht. Paderborn. http://www.evb-online.de/evb_revis.php

Schlegel-Matthies, K., Bartsch, S., Brandl, W., Heindl, I. \& Methfessel, B. (in Vorbereitung). Studienbuch Konsum - Ernährung - Gesundheit.

Schönberger, G. (2005). Sinne und Sensorik, Essen und Ambiente. In D. von Engelhardt, R. Wild \& G. Neumann (Hrsg.), Geschmackskulturen. Vom Dialog der Sinne beim Essen und Trinken (S. 34-46). Frankfurt a. M.: Campus.

Schulz, B. (2012). Von der Lust zum Genuss. Genussbildung mit Jugendlichen. GUT DRAUF Infomagazin, 4, 2-4.

https://www.gutdrauf.net/fileadmin/user_upload/Startseite/Materialien/GDInfomagazin/GD_Infomagazin_04_12.pdf

Spitzer, M. (2006). Medizin für die Schule. Plädoyer für eine evidenzbasierte Pädagogik. In R. Caspary (Hrsg.), Lernen und Gehirn. Der Weg zu einer neuen Pädagogik (2. Aufl., S. 23-35). Freiburg im Breisgau: Herder.

Warburton, D. M. (2000). Wohlbefinden, Ernährung und Freude. Internationaler Arbeitskreis für Kulturforschung des Essens, 5, 24-30.

Wierlacher, A. (2008). Oralität und Kulturalität von Geschmack und Genuss. In A. Wierlacher \& R. Bendix (Hrsg.), Kulinaristik. Forschung - Lehre - Praxis (S. 157-171). Berlin: Lit.

\section{Verfasserin}

Barbara Rieger M.A.

Pädagogische Hochschule Karlsruhe

Bismarckstraße 10

D-76133 Karlsruhe

E-Mail: b-rieger@web.de 